\title{
Mitochondria-targeted antioxidant attenuates high glucose-induced P38 MAPK pathway activation in human neuroblastoma cells
}

\author{
MINGFENG CAO $^{1 *}$, JINJIAO JIANG $^{1 *}$, YIFENG DU $^{2}$ and PENG YAN ${ }^{2}$ \\ Departments of ${ }^{1}$ Endocrinology and ${ }^{2}$ Neurology, Provincial Hospital Affiliated \\ to Shandong University, Jinan 250021, P.R. China
}

Received September 28, 2011; Accepted December 27, 2011

DOI: $10.3892 / \mathrm{mmr} .2012 .746$

\begin{abstract}
Excessive mitochondrial free radical production and the related mitogen-activated protein kinase P38 (P38 MAPK) activation are key regulators in the pathogenesis of high glucose-induced cell stress. Increasing evidence has emphasized the impact of hyperglycemia on neurons and the consequent neuronal stresses eventually resulting in neurodegeneration and neuronal death. In this study, we employed a novel mitochondria-targeted antioxidant, SS31 peptide, on high glucose-insulted neuroblastoma cells (SH-SY5Y). Our results showed that high glucose promoted significantly increased P38 phosphorylation which was efficiently suppressed by the application of the SS31 peptide under the experimental conditions. The inhibition of high glucose-induced P38 activation by the SS31 peptide was associated with the impact of the SS31 peptide on attenuating high glucose-induced mitochondrial ROS (reactive oxygen species) elevation and mitochondrial membrane potential collapse. The addition of SS31 peptide significantly attenuated high-gluose-induced apoptosis. Therefore, our study suggests that elimination of high glucose-induced mitochondrial oxidative stress helps to rescue SH-SY5Y cells from high glucose-related P38 MAPK pathway disturbances, and the SS31 peptide has the potential to serve as a new treatment strategy against hyperglycemia-instigated neuronal perturbations.
\end{abstract}

\section{Introduction}

Dysregulated glucose metabolism underlies a variety of pathologies leading to impairments in multiple types of cells

Correspondence to: Dr Jinjiao Jiang, Department of Endocrinology Provincial Hospital Affiliated to Shandong University, Jinan 250021, P.R. China

E-mail: jjjsch@sina.com

Dr Yifeng Du, Department of Neurology, Provincial Hospital Affiliated to Shandong University, Jinan 250021, P.R. China E-mail: syrsc97@163.com

Key words: high-glucose, SS31 peptide, antioxidant, mitogenactivated protein kinase $\mathrm{P} 38$, mitochondrial reactive oxygen species, neuroblastoma cell including neurons (1). Recently, emerging evidence has accentuated the impact of high blood glucose on neuronal function in vitro and in vivo suggesting the correlation between glucose dysmetabolism and diverse types of brain diseases including stroke and neurodegenerative diseases $(1,2)$. Mitochondria are the main glucose-consuming sites in neurons and produce ATP by glycolysis through oxidation phosphorylation. Previous studies have revealed that high blood glucose substantially encumbers mitochondrial function and results in mitochondria-dependent apoptosis suggesting mitochondrial pathology stands in the nexus of glucose dysmetabolism and cell injuries $(3,4)$. Among the many glucose dysmetabolism-induced mitochondrial dysfunctions, increased mitochondria oxidative stress is a predominant mitochondrial pathology and occurs early in response to high-glucose insult (5). Correspondingly, it has been widely reported that excessive reactive oxygen species (ROS) plays a key role in the pathogenesis of high glucoseassociated pathological conditions such as neurodegeneration and diabetes $(5,6)$. Oxidative stress is a strong inducer of the mitogen-activated protein kinase P38 (P38 MAPK) activation (7) and the activation of P38 MAPK signaling pathway is another prominent change in high glucose-exposed cells. The excessive activation of P38 MAPK is a critical mechanism underlying high glucose-induced cellular injuries eventually leading to apoptosis $(8,9)$. Thus, elimination of high glucoseinduced mitochondrial ROS may have the potential to attenuate P38 MAPK signaling disturbances.

The SS31 peptide (H-D-Arg-Dmt-Lys-Phe-NH2) is a novel cell permeable antioxidant specifically targeted to mitochondria and subsequently eliminates mitochondrial reactive oxygen species (ROS) and preserves ATP production against oxidizing insults $(10,11)$. Yet, to the best of our knowledge, the effect of the SS31 peptide on P38 MAPK signaling perturbation in high glucose-insulted neurons has not yet been elucidated. In the present study, using a neuron-like cell line (human neuroblastoma SH-SY5Y) we demonstrated that the use of the SS31 peptide substantially suppressed high glucoseinstigated mitochondrial oxidative stress and P38 MAPK activation in neuroblastoma cells which serves as evidence of the potential application of mitochondria-targeted antioxidants in the treatment of glucose dysmetabolism-induced neuronal stresses. 


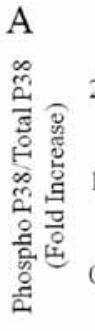

C
B

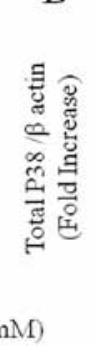

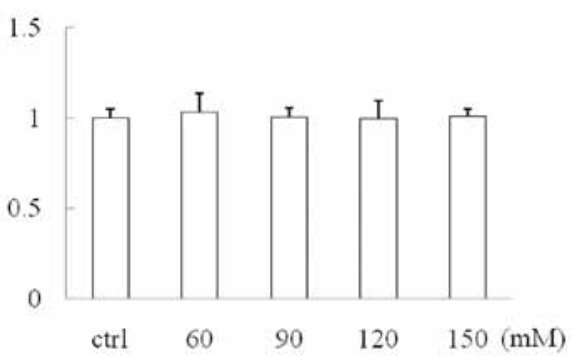

Phospho P38

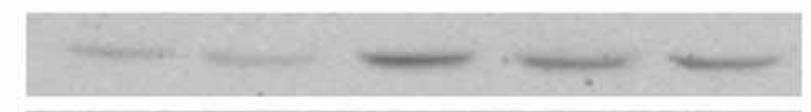

Total P38

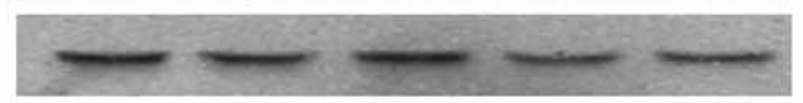

$\beta$ actin

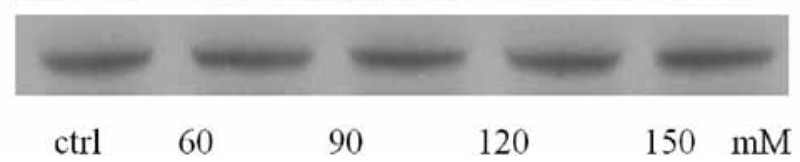

Figure 1. Cells were exposed to glucose at the indicated concentrations for 90 min and then subjected to western blotting. The P38 phosphorylation level was significantly increased in cells exposed to (A) high concentration of glucose while (B) the total P38 level remained the same. (C) Representative immunoreactive bands of phospho P38, total P38 and $\beta$-actin. ${ }^{*} \mathrm{P}<0.05$ vs. the control group. Data are derived from 3 independent experiments.

\section{Materials and methods}

Cell culture and treatment. Human neuroblastoma cells (SH-SY5Y) were obtained from the American Type Culture Collection (ATCC). Cells were cultured in DMEM $(25 \mathrm{mM}$ glucose, Gibco, USA) with $5 \%$ fetal bovine serum (Gibco), $2 \mathrm{mM}$ L-glutamine, $100 \mathrm{U} / \mathrm{ml}$ penicillin in a $95 \%$ air, $5 \% \mathrm{CO}_{2}$ atmosphere. Cells were starved $12 \mathrm{~h}$ before the experiments by replacing the medium with OptiMEM (Gibco) without fetal bovine serum, followed by treatment with D-glucose (Sigma) as vehicle (no additional glucose added) at indicated final concentrations. The SS31 peptide (Stealth Peptide International, China) at the indicated concentration was applied $30 \mathrm{~min}$ before the addition of glucose. Antimycin A (Sigma) treatment was conducted at $50 \mu \mathrm{M}$ on cells for $2 \mathrm{~h}$. Carbonylcyanide p-trifluoromethoxyphenylhydrazone (FCCP; Sigma) was used at $5 \mathrm{mM}$ to treat cells for $90 \mathrm{~min}$.

Intracellular and intra-mitochondrial ROS and mitochondrial membrane potential detection. Intracellular ROS was detected by using fluorescence probe 5-(and 6-) chloromethyl-20, 70-dichlorodihydrofluorescein diacetate (CM-DCF-DA; Invitrogen, USA) at $10 \mu \mathrm{M}$ for $30 \mathrm{~min}$, and intra-mitochondrial ROS was visualized by coincubation of MitoSox Red (Invitrogen) at $1 \mu \mathrm{M}$. To detect mitochondrial membrane potential, tetramethylrhodamine methyl ester (TMRM; Invitrogen) was used at $200 \mathrm{nM}$ for $20 \mathrm{~min}$ followed by washing using culture medium. Cells, after appropriate staining were observed under a Zeiss Axiovert 200M inverted fluores,cence microscope with heating control and $\mathrm{CO}_{2}$ chamber. The intensity of fluorescent staining was calculated as (F1-F0). F1 is the intensity of the staining and F0 is the intensity of the background.

Western blotting. The proper amount of cell extracts was loaded and separated by SDS-PAGE and transferred to nitrocellulose membranes (Bio-Rad, USA). Immunoblotting was performed using antibodies against phospho P38 (pT180/ pY182, BD; 1:1,000), P38 (Cell Signaling, USA; 1:3,000) or $\beta$-actin (Sigma; $1: 5,000$ ) overnight at $4^{\circ} \mathrm{C}$. Immunoreactive bands were visualized using the ECL system (Fisher Scientific, USA).

TUNEL assay. Cell apoptosis was measured by terminal deoxynucleotidyl transferase dUTP nick end labeling (TUNEL) method using the TUNEL Assay kit (Roche, Germany) following the manufacturer's instructions.

Statistics and data analysis. Statistical analysis was performed by one-way ANOVA and post-hoc ANOVA analysis when appropriate using SPSS software. The values are shown as mean \pm SEM. Significance was defined as $\mathrm{P}<0.05$. Analysis of immunoblotting and cell staining was conducted using $\mathrm{NIH}$ Image J software (1.62).

\section{Results}

High glucose induces P38 MAPK activation in SH-SY5Y cells in a dose-dependent manner. Cells were exposed to vehicle, $60,90,120$ and $150 \mathrm{mM}$ glucose for $90 \mathrm{~min}$ after starvation and then subjected to immunoblotting to detect the levels of phospho P38 and total P38 MAPK. $\beta$-actin was adopted as the loading control to verify that the same amount of proteins was loaded in each well. Our data showed that the P38 phosphorylation level was increased in a dose-dependent manner with increased glucose concentration. Glucose treatment $(60 \mathrm{mM})$ induced a mild increase in P38 phosphorylation as compared with the vehicle group (Fig. 1A, P>0.05); whereas glucose at concentrations of $90 \mathrm{mM}$ or higher significantly instigated P38 phosphorylation in the treated cells (Fig. 1A, P<0.05 vs. control group). In contrast, the level of total P38 was not changed in any group by comparison with $\beta$-actin suggesting 
A

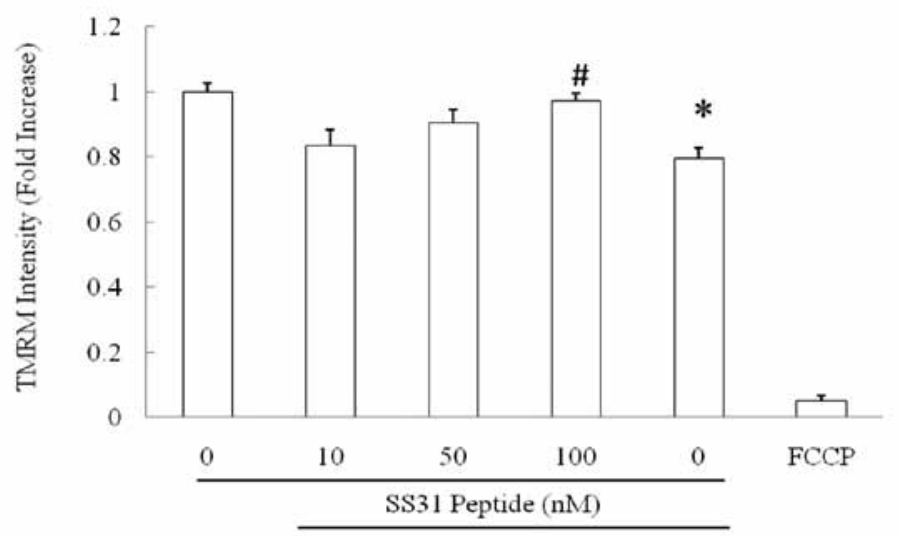

B
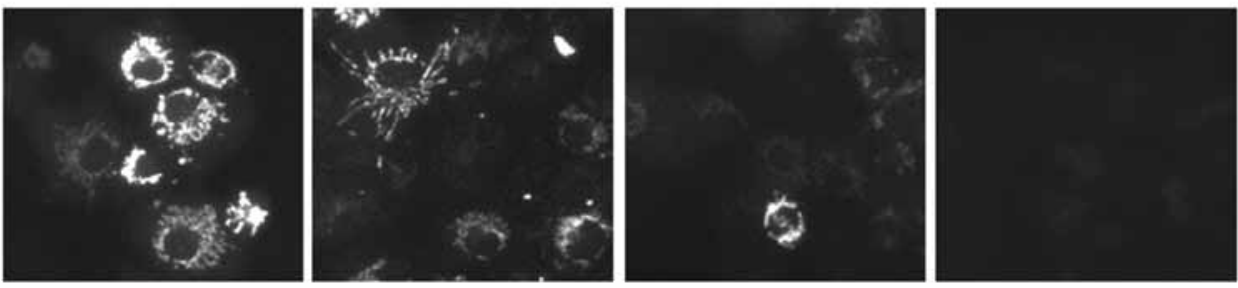

ctrl

SS31 $100 \mathrm{nM}+$ Glucose

Glucose

FCCP

Figure 2. SH-SY5Y cells were exposed to the SS31 peptide at 10,50 and $100 \mathrm{nM}$ for $30 \mathrm{~min}$ followed by the addition of glucose (final concentration $90 \mathrm{mM}$ ). FCCP was used as a positive control. (A) SS31 peptide significantly preserved mitochondrial membrane potential from high-glucose insult in a dose-dependent manner. ${ }^{*} \mathrm{P}<0.05$ vs. control group and ${ }^{~} \mathrm{P}<0.05$ vs. high-glucose group. (B) Representative images of TMRM. Data are derived from 3 independent experiments.

that the increased P38 phosphorylation in the glucose treatedgroups was not due to an increased level of total P38.

SS31 peptide protects mitochondrial membrane potential against high-glucose insult. Mitochondrial membrane potential is a critical indicator of mitochondrial function, and decreased mitochondrial membrane potential is closely associated with elevated mitochondrial ROS production. Previous studies have shown that the SS31 peptide confers substantial protection on mitochondrial membrane potential against multiple insults $(3,12,13)$. We next studied whether the SS31 peptide preserves mitochondrial membrane potential against high glucose in SH-SY5Y cells. FCCP, a strong mitochondrial uncoupler, was employed to treat cells at $5 \mathrm{mM}$ as a positive control. High glucose substantially decreased mitochondrial membrane potential to $79.5 \%$ in comparison to the vehicle group (Fig. 2, $\mathrm{P}<0.0001)$; whereas the adoption of the SS31 peptide demonstrated a dose-dependent rescue of the high glucose-induced mitochondrial membrane potential collapse. Of note, the SS31 peptide at the concentration of $100 \mathrm{nM}$ exhibited significant restoration of high glucose-insulted mitochondrial membrane potential decrease almost to the same level as that in the vehicle group (Fig. 2, vs. high glucose-treated group; $\mathrm{P}<0.0001$ ); while the SS31 peptide at $50 \mathrm{nM}$ also showed partial recovery as compare to the high glucose-treated group (Fig. 2A, $\mathrm{P}<0.05$ ). FCCP-exposed cells demonstrated significantly lower mitochondrial membrane potential indicating the specificity of TMRM as a mitochondrial membrane potential indicator.

SS31 peptide suppresses high glucose-induced intracellular and intra-mitochondrial ROS production. Mitochondrial membrane potential collapse is closely associated with mito- chondrial ROS production. As the SS31 peptide at $100 \mathrm{nM}$ significantly restored mitochondrial membrane potential in SH-SY5Y cells against high-glucose insult, we next observed the impact of the SS31 peptide at $100 \mathrm{nM}$ on high glucoseinduced cellular and mitochondrial ROS production. We compared the intracellular and intra-mitochondria ROS levels between the vehicle- and glucose-treated groups. Intracellular ROS was detected using DCF-DA and intra-mitochondrial ROS was shown as the increased intensity of MitoSox Red, a specific mitochondrial ROS indicator. The data showed that cells exposed to $90 \mathrm{mM}$ glucose for $90 \mathrm{~min}$ underwent significantly increased intra-mitochondrial and intracellular ROS levels by $40.5 \%$ (Fig. 3A and C, $\mathrm{P}<0.0001$ ) and $45.5 \%$ (Fig. 3B and C, $\mathrm{P}=0.0047$ ), respectively, as compared with the vehicle group. In sharp contrast, the addition of the SS31 peptide significantly suppressed the high glucose-induced increase in intracellular and intra-mitochondrial ROS levels. In the SS31 peptide-treated group, the intra-mitochondrial ROS level was reduced by $28.0 \%$ (Fig. $3 \mathrm{~A}$ and $\mathrm{C}, \mathrm{P}=0.0008$ ) while intracellular ROS level was decreased by $26.6 \%$ (Fig. $3 \mathrm{~B}$ and $\mathrm{C}$, $\mathrm{P}=0.0359$ ) in comparison with the high glucose-treated group implicating the effect of SS31 peptide application on high glucose-instigated ROS generation.

Elevated mitochondrial ROS production instigates P38 MAPK activation in SH-SY5Y cells. Oxidative stress is a known causative factor of P38 activation. Mitochondria are the major sites of ROS production. In order to examine whether P38 activation is related to mitochondrial ROS production in SH-SY5Y cells, we applied antimycin A to cultured cells. Antimycin A is the inhibitor of mitochondrial complex III and is a strong inducer of mitochondrial ROS production. Cells were exposed 

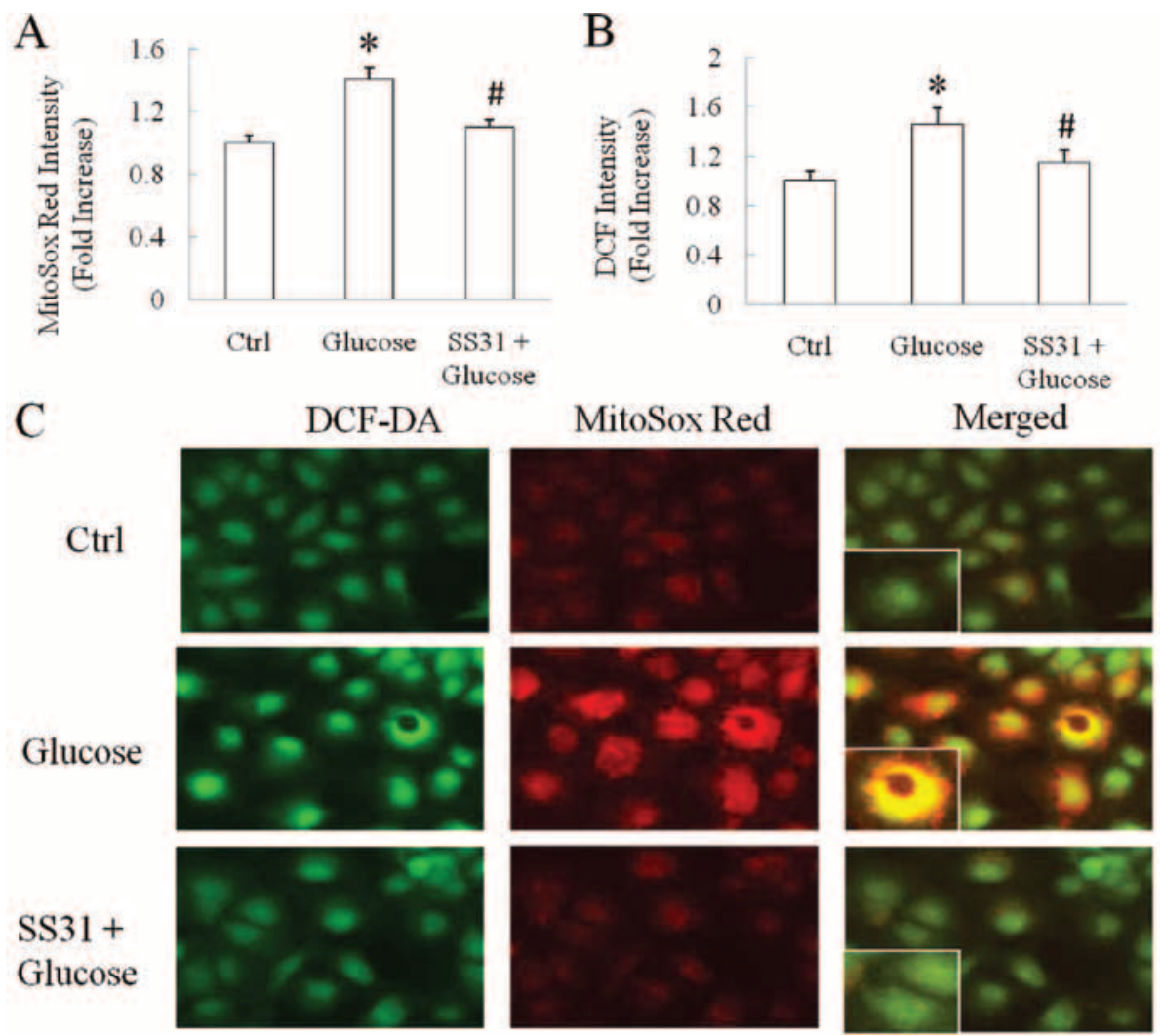

Figure 3. SH-SY5Y cells were exposed to SS31 peptide $100 \mathrm{nM}$ for $30 \mathrm{~min}$ followed by $90 \mathrm{mM}$ glucose for $90 \mathrm{~min}$. The SS31 peptide significantly quenched the (A) mitochondrial ROS and (B) high glucose-induced cellular levels. (A) " $\mathrm{P}<0.0001$ vs. control group and ${ }^{*} \mathrm{P}<0.05$ vs. high-glucose group. (B) " $\mathrm{P}=0.0047$ vs. control group and ${ }^{\#} \mathrm{P}<0.05$ vs. high-glucose group. (C) Representative images of DCF-DA (green) and MitoSox Red (red) staining in indicated groups. Inserted images are enlarged from the original images. Data are derived from 3 independent experiments and shown as fold increase.

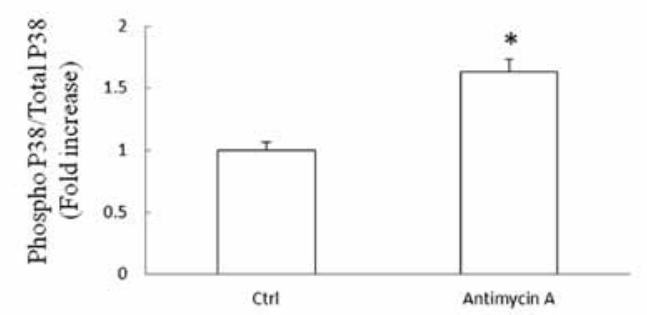

Phospho P38

Total P38

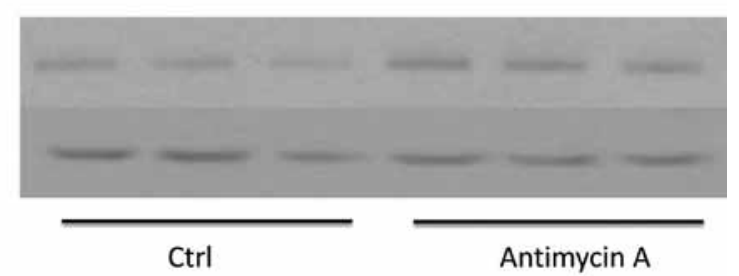

Figure 4. SH-SY5Y cells were exposed to $50 \mu \mathrm{M}$ antimycin A for $2 \mathrm{~h}$ and then subjected to immunoblotting for phospho P38 and total P38. Antimycin A induced a 63\% increase in P38 activation as compared to the vehicle control (upper panel); " $\mathrm{P}<0.05$. The lower panel shows representative immunoreactive bands. Data are derived from 3 independent experiments.

to antimycin $\mathrm{A}$ at $50 \mu \mathrm{M}$ for $2 \mathrm{~h}$ and subjected to immunoblotting to detect the P38 phosphorylation level. The ratio of phospho P38 to total P38 was employed as the manifestation of P38 activation. Our data showed that antimycin A induced a $63 \%$ increase in P38 activation level in comparison to the control (Fig. 4, $\mathrm{P}<0.05$ ). The result indicates that mitochondrial ROS production/release is associated with P38 activation in SH-SY5Y cells.
SS31 peptide inhibits high glucose-induced P38 phosphorylation in SH-SY5Y cells in a dose-dependent manner. Finally, we determined whether suppressed mitochondrial ROS subsequently inhibits high glucose-instigated P38 activation. We employed the SS31 peptide at 0, 10, 50100 and $200 \mathrm{nM}$, respectively. Cells were exposed to the SS31 peptide at indicated concentrations for $30 \mathrm{~min}$ followed by incubation with glucose at $90 \mathrm{mM}$ for $90 \mathrm{~min}$. The coincubation of the SS31 
A

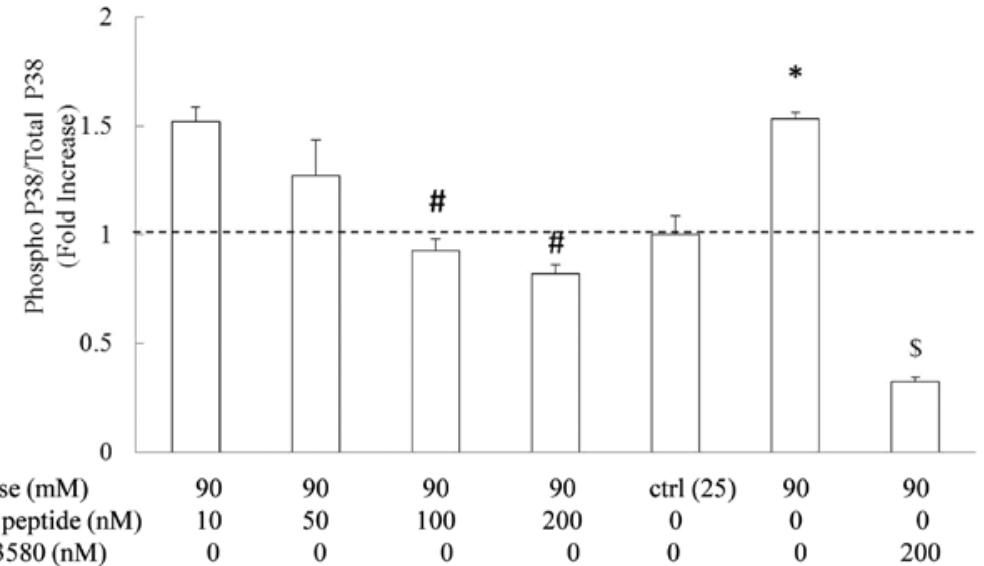

B

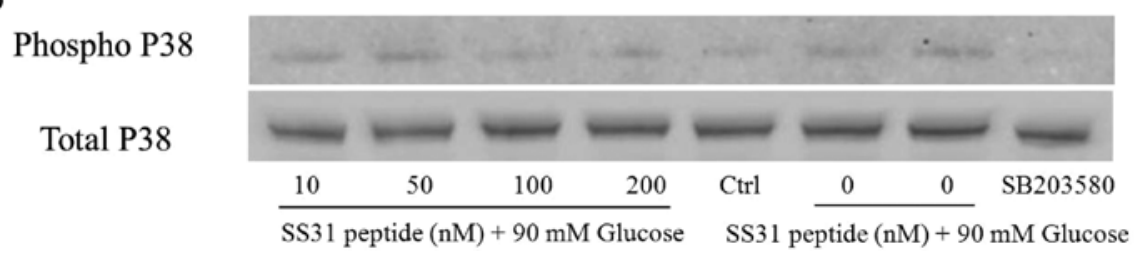

Figure 5. (A) SS31 peptide demonstrated a dose-dependent inhibition of high glucose-induced P38 activation. (B) Representative immunoreactive bands of phospho P38 and total P38. ${ }^{*} \mathrm{P}<0.05$ vs. the control group and $50 \mathrm{nM} \mathrm{SS} 31$ peptide group; ${ }^{\#} \mathrm{P}<0.05$ vs. high glucose-treated group; ${ }^{{ }^{S}} \mathrm{P}<0.05$ vs. all the other groups. Data are derived from 3 independent experiments.

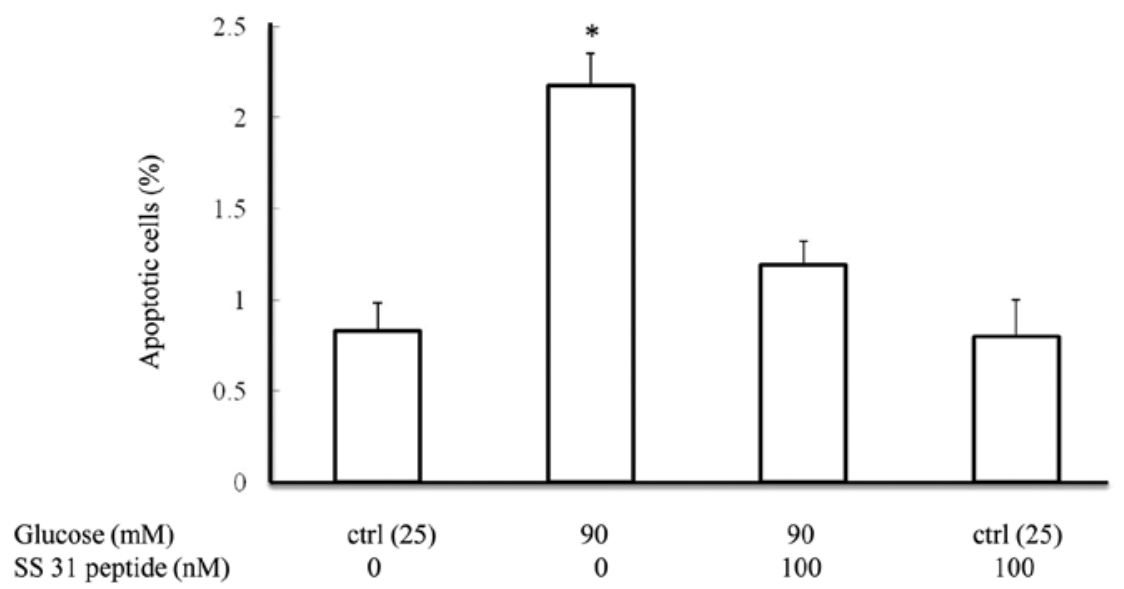

Figure 6. SS31 peptide attenuates high glucose-induced apoptosis. Glucose $(90 \mathrm{mM})$ induced increased apoptosis in SH-SY5Y cell after 24-h treatment which was significantly ameliorated by the SS31 peptide $(100 \mathrm{nM})$. ${ }^{*} \mathrm{P}<0.05$ vs. other groups.

peptide with high glucose markedly attenuated high glucoseinduced P38 activation in a dose-dependent manner. As shown in Fig. 5, high-glucose treatment increased the P38 phosphorylation level to $2.33 \pm 0.14$-fold as compared with the vehicle group (Fig. 5A, $\mathrm{P}<0.05$ ). In sharp contrast, the application of the SS31 peptide at 100 and $200 \mathrm{nM}$ substantially decreased P38 phosphorylation to a similar level in comparison with that in the vehicle control ( $\mathrm{P}>0.05$ vs. vehicle group), which was significantly lower than that in the high glucose-treated group (Fig. 5A, P<0.05). Although the SS31 peptide at $10 \mathrm{nM}$ exhibited limited inhibition on high glucose-induced $\mathrm{P} 38$ activation, the SS31 peptide at $50 \mathrm{nM}$ demonstrated partial protection on high glucose-induced $\mathrm{P} 38$ activation (Fig. 5A, $\mathrm{P}<0.05$ ). The dose-dependent inhibition of the SS31 peptide on glucose- induced P38 activation was in correlation with the impact of the SS31 peptide on high glucose-instigated mitochondrial membrane potential and ROS production perturbations. The addition of P38 inhibitor SB203580 (200 nM) significantly suppressed high glucose-induced P38 activation (Fig. 5A, $\mathrm{P}<0.05$ vs. high glucose-treated group). Thus, our data indicate a protective effect of the SS31 peptide on P38 activation in SH-SY5Y cells against high-glucose exposure.

SS31 peptide attenuates high glucose-induced SH-SY5Y cell apoptosis. P38 activation has been implicated to be involved in apoptosis in response to cell injuries. We then assessed the protection of the SS31 peptide on apoptosis in the face of high-glucose insult. SH-SY5Y cells were exposed to $90 \mathrm{mM}$ 
glucose in the presence or absence of $100 \mathrm{nM}$ SS31 peptide for $24 \mathrm{~h}$ and then subjected to TUNEL assay. Our data showed that high glucose-induced a 2.6 -fold increase in the percentage of apoptotic cells while the application of the SS31 peptide significantly ameliorated the high glucose-induced apoptosis (Fig. $6, \mathrm{P}<0.05$ vs. other groups). The SS31 peptide alone did not have a significant effect on cell apoptosis.

\section{Discussion}

Hyperglycemia is a potential culprit underlying peripheral neuron changes in diabetes and neurodegeneration in the central nervous system (CNS) $(6,14)$. The P38 MAPK signaling pathway plays a key role in controlling cell proliferation while excessive P38 activation leads to mitochondria-dependent apoptosis. It should be noted that P38 MAPK signaling activation is a predominant cell change in response to high-glucose insult and is significantly involved in high glucose-related cell injuries including decreased mitochondrial function and mitochondria-dependent apoptosis in the pathogenesis of hyperglycemia-associated diseases $(9,15)$. Previous studies have shown that mitochondrial ROS is an initiator of P38 activation in cardiomyocytes and neurons under hypoxia and hyperglycemic conditions $(16,17)$. The elimination of excessive cellular ROS has the potential to protect against high glucose-induced P38 activation. Indeed, various ROS scavengers such as VitE, SOD and lipid acid have been applied to treat hyperglycemiaassociated cell stresses. However, the applications of these antioxidants are limited by multiple drawbacks such as high drug dose and various side effects (18-20). The SS31 peptide is a novel antioxidant specifically targeted to mitochondria and efficiently quenches mitochondrial ROS in many pathological conditions. Importantly, its relatively small-molecular weight and lipid solubility enable the peptide to rapidly penetrate cells (21). Our result showed that the addition of the SS31 peptide significantly reversed the high glucose-induced mitochondrial ROS elevation and mitochondrial membrane potential collapse. These findings are in agreement with many previous studies on the pharmaceutical effects of the SS31 peptide $(10,13)$. Furthermore, by eliminating mitochondrial ROS and protecting mitochondrial membrane potential, P38 activation as well as apoptosis in high glucose-treated SH-SY5Y cells was substantially suppressed by the application of the SS31 peptide. The SS31 peptide protects cells from high-glucose insult. Notably, we adopted a neuron-like cell line, SH-SY5Y which demonstrates neuron biological features. To the best of our knowledge, to date, reports are limited on the effect of SS31 peptide against high glucose-induced mitochondrial ROS as well as neuronal dysfunctions. These results serve as important evidence for the application of this peptide in preventing neuronal stresses and injury related to hyperglycemia.

Collectively, we demonstrated that the nascent mitochondria-targeted antioxidant, SS31 peptide, substantially rescues high glucose-associated mitochondrial dysfunction and P38 activation in SH-SY5Y cells. This provides evidence that the SS31 peptide is a promising treatment strategy for rescuing neuronal function from hyperglycemia. Moreover, the small size of the SS31 peptide and its lipid solubility substantially enable the use of the SS31 peptide in treating hyperglycemiainstigated neuronal stresses in the CNS.

\section{Acknowledgements}

This study was supported by a grant from the Science Foundation of Shandong Province (Grant No. Q2006C15), China.

\section{References}

1. Das F, Dey N, Venkatesan B, Kasinath BS, Ghosh-Choudhury N and Choudhury GG: High-glucose upregulation of early-onset Parkinson's disease protein DJ-1 integrates the PRAS40/TORC1 axis to mesangial cell hypertrophy. Cell Signal 23: 1311-1319, 2011.

2. Matsuzaki T, Sasaki K, Tanizaki Y, et al: Insulin resistance is associated with the pathology of Alzheimer disease: the Hisayama study. Neurology 75: 764-770, 2010.

3. Li J, Chen X, Xiao W, et al: Mitochondria-targeted antioxidant peptide SS31 attenuates high-glucose-induced injury on human retinal endothelial cells. Biochem Biophys Res Commun 404: 349-356, 2011.

4. Schiff M, Loublier S, Coulibaly A, Benit P, de Baulny HO and Rustin P: Mitochondria and diabetes mellitus: untangling a conflictive relationship? J Inher Metab Dis 32: 684-698, 2009.

5. Herlein JA, Fink BD and Sivitz WI: Superoxide production by mitochondria of insulin-sensitive tissues: mechanistic differences and effect of early diabetes. Metabolism 59: 247-257, 2010.

6. Wang HL, Chou AH, Wu AS, et al: PARK6 PINK1 mutants are defective in maintaining mitochondrial membrane potential and inhibiting ROS formation of substantia nigra dopaminergic neurons. Biochimic Biophys Acta 1812: 674-684, 2011.

7. Ravindran J, Gupta N, Agrawal M, Bala Bhaskar AS and Lakshmana Rao PV: Modulation of ROS/MAPK signaling pathways by okadaic acid leads to cell death via mitochondrial-mediated caspase-dependent mechanism. Apoptosis 16: 145-161, 2011.

8. Begum N and Ragolia L: High-glucose and insulin inhibit VSMC MKP-1 expression by blocking iNOS via P38 MAPK activation. Am J Physiol Cell Physiol 278: C81-C91, 2000.

9. Ren Y, Shi Y, Wang Y, et al: P38 MAPK pathway is involved in high-glucose-induced thioredoxin interacting protein induction in mouse mesangial cells. FEBS Lett 584: 3480-3485, 2010.

10. Carter EA, Bonab AA, Goverman J, et al: Evaluation of the antioxidant peptide SS31 for treatment of burn-induced insulin resistance. Int J Mol Med 28: 589-594, 2011.

11. Reddy TP, Manczak M, Calkins MJ, et al: Toxicity of neurons treated with herbicides and neuroprotection by mitochondriatargeted antioxidant SS31. Inter J Environ Res Public Health 8: 203-221, 2011.

12. Andersson DC, Fauconnier J, Yamada T, et al: Mitochondrial production of reactive oxygen species contributes to the betaadrenergic stimulation of mouse cardiomycytes. J Physiol 589: 1791-1801, 2011.

13. Manczak M, Mao P, Calkins MJ, et al: Mitochondria-targeted antioxidants protect against amyloid-beta toxicity in Alzheimer's disease neurons. J Alzheimers Dis 20 (Suppl 2): S609-S631, 2010.

14. Toth C, Brussee V, Cheng C and Zochodne DW: Diabetes mellitus and the sensory neuron. J Neuropathol Exp Neurol 63: 561-573, 2004.

15. Wilmer WA, Dixon CL and Hebert C: Chronic exposure of human mesangial cells to high-glucose environments activates the P38 MAPK pathway. Kidney Int 60: 858-871, 2001.

16. Kulisz A, Chen N, Chandel NS, Shao Z and Schumacker PT: Mitochondrial ROS initiate phosphorylation of p38 MAP kinase during hypoxia in cardiomyocytes. Am J Physiol Lung Cell Mol Physiol 282: L1324-L1329, 2002.

17. Sharma R, Buras E, Terashima T, et al: Hyperglycemia induces oxidative stress and impairs axonal transport rates in mice. PloS One 5: e13463, 2010.

18. Brownlee M: The pathobiology of diabetic complications: a unifying mechanism. Diabetes 54: 1615-1625, 2005.

19. Guerrero-Romero F and Rodriguez-Moran M: Complementary therapies for diabetes: the case for chromium, magnesium, and antioxidants. Arch Med Res 36: 250-257, 2005.

20. Vincent AM, Russell JW, Low P and Feldman EL: Oxidative stress in the pathogenesis of diabetic neuropathy. Endocr Rev 25: 612-628, 2004.

21. Cho S, Szeto HH, Kim E, Kim H, Tolhurst AT and Pinto JT: A novel cell-permeable antioxidant peptide, SS31, attenuates ischemic brain injury by down-regulating CD36. J Biol Chem 282: 4634-4642, 2007. 